\title{
The Gamow-Teller Resonance in Finite Nuclei in the Relativistic Random Phase Approximation
}

\author{
Zhong-Yu Ma ${ }^{1,2}$, Bao-Qiu Chen ${ }^{1,2}$, Nguyen Van Giai ${ }^{3}$, and Toshio Suzuki ${ }^{4}$ \\ 1 China Institute of Atomic Energy, Beijing 102413 \\ ${ }^{2}$ Center of Theoretical Nuclear Physics, National Laboratory \\ of Heavy Ion Accelerator of Lanzhou, Lanzhou 730000 \\ ${ }^{3}$ Institut de Physique Nucléaire, IN2P3-CNRS, F-91406 Orsay Cedex, France \\ ${ }^{4}$ Department of Applied Physics, Fukui University, Fukui 910-8507, \\ Japan and RIKEN, 2-1 Hirosawa, Wako-shi, Saitama 351-0198, Japan \\ Gamow-Teller(GT) resonances in finite nuclei are studied in a fully consistent \\ relativistic random phase approximation (RPA) framework. A relativistic form of \\ the Landau-Migdal contact interaction in the spin-isospin channel is adopted. This \\ choice ensures that the GT excitation energy in nuclear matter is correctly repro- \\ duced in the non-relativistic limit. The GT response functions of doubly magic nuclei \\ ${ }^{48} \mathrm{Ca},{ }^{90} \mathrm{Zr}$ and ${ }^{208} \mathrm{~Pb}$ are calculated using the parameter set NL3 and $g_{0}^{\prime}=0.6$. It is \\ found that effects related to Dirac sea states account for a reduction of $6-7 \%$ in the \\ GT sum rule. \\ PACS numbers: 21.60.-n, 21.60.Jz, 24.10.Jv, 24.30.Cz
}

In recent years, the relativistic mean field (RMF) theory with non-linear meson selfinteractions has achieved a great success in describing bulk properties of nuclei, not only spherical but also deformed nuclei and nuclei far from the $\beta$-stability line 1]. In particular, a fully consistent relativistic random phase approximation (RPA) based on the RMF has been established [2, 3, 4, 5]. The consistency implies that the particle-hole (p-h) residual interaction and the nuclear mean field are calculated from the same effective Lagrangian. The relativistic RPA is equivalent to the time-dependent RMF in the small amplitude limit [6, 7] only if the particle-hole configuration space includes not only the pairs formed from the occupied and unoccupied Fermi states but also the pairs formed from the Dirac states and occupied Fermi states [8]. It has been found that the effective Lagrangians which can well describe the ground state properties of nuclei could also reproduce their collective excited states and giant resonances [9].

The investigation of nuclear excitations involving spin-isospin Gamow-Teller(GT) resonances 
has attracted a great interests for a long time. The nuclear GT transitions are essential to many important processes in particle physics and astrophysics related to neutrino-nucleus interactions. The quenching of the observed GT strength in nuclei is a long standing issue both experimentally and theoretically. It has been advanced through the recent extensive investigations of GT resonances by the charge exchange reactions at intermediate energies [10, 11]. The recent $(p, n)$ and $(n, p)$ measurements have observed about $90 \%$ of the Ikeda sum rule[12] at energies $E_{x}<50$ $\mathrm{MeV}$ in medium heavy nuclei[11]. Theoretical investigations have shown that there are several possible mechanisms for the unseen strength, which range from the admixture of $2 p-2 h$ components in even-even nuclei [13] to the coupling to the $\Delta$-hole sector [14]. The theoretical study of GT matrix elements in nuclei has already revealed some valuable information of nuclear structure effects and non-nucleonic degrees of freedom such as mesons and delta-isobars. A further and clear understanding of the GT resonances is still required. Up to now most investigations in the relativistic approach are restricted to the electric excitations. Although the GT resonance has been recently studied in the relativistic RPA [15, 16] a proper relativistic form of the p-h interaction in the spin-isospin channel has not been obtained.

A relativistic form of the Landau-Migdal $g^{\prime}$ term which could reproduce the non-relativistic result for the GT excitation energy in nuclear matter has been recently proposed[17]. The study of the relativistic description of GT resonances in nuclear matter has pointed out a new quenching mechanism due to the relativistic effects. The purpose of this letter is to investigate the GT resonances in finite nuclei in the fully consistent relativistic RPA. The GT response functions of ${ }^{208} \mathrm{~Pb},{ }^{90} \mathrm{Zr}$ and ${ }^{48} \mathrm{Ca}$ are studied. We shall emphasize the contribution of the Dirac states to the quenching of the Ikeda sum rule in the GT strengths due to the completeness of nuclear wave functions as pointed out for nuclear matter in ref.[17].

The relativistic RPA is built on the ground state which is calculated in the RMF theory. For a consistent RPA calculation the residual p-h interaction giving rise to giant resonances must be obtained from the same Lagrangian. The spin-isospin correlations in the relativistic approach are induced by the isovector mesons $\pi$ and $\rho$. In the existing effective Lagrangians explicitly built for RMF the pion term does not appear since it does not contribute to the mean field while for the $\rho$ the tensor coupling term is generally ignored and the vector coupling term is strongly renormalized to give the correct neutron-proton symmetry energy. To remain consistent we choose to introduce in the effective Lagrangian a pseudo-vector pion term but no $\rho$ tensor term. Thus, the GT correlations will be induced by the Lagrangian

$$
\mathcal{L}=-\frac{f_{\pi}}{m_{\pi}} \bar{\psi} \gamma_{5} \gamma^{\mu} \partial_{\mu} \boldsymbol{\tau} \cdot \boldsymbol{\pi} \psi-g_{\rho} \bar{\psi} \gamma_{\mu} \partial_{\mu} \boldsymbol{\tau} \cdot \boldsymbol{\rho}^{\mu} \psi
$$


It is well known that due to the pseudo-vector coupling of the pion and tensor coupling of the $\rho$ an effective interaction with the Landau-Migdal parameter $g^{\prime}$ term has to be introduced, which has the form $g^{\prime} \boldsymbol{\sigma}_{1} \cdot \boldsymbol{\sigma}_{2} \boldsymbol{\tau}_{1} \cdot \boldsymbol{\tau}_{2}$ in the non-relativistic limit. There are several ways to introduce this term in the interaction Lagrangian [15, 18]. However, It has been pointed out in Ref. 17] that the way to introduce $g^{\prime}$ in the relativistic model is model-dependent and that the following choice

$$
\mathcal{L}=\frac{g_{5}}{2} \bar{\psi} \Gamma_{i}^{\mu} \psi \bar{\psi} \Gamma_{\mu i} \psi, \quad \Gamma_{i}^{\mu}=\gamma_{5} \gamma^{\mu} \tau_{i}, \quad g_{5}=g^{\prime} \frac{f_{\pi}^{2}}{m_{\pi}^{2}},
$$

insures that, in the non-relativistic limit one can recover the expression of the GT excitation energy in nuclear matter.

The response function of the system to an external field is given by the imaginary part of the retarded polarization operator,

$$
R\left(P, P ; k, k^{\prime} ; E\right)=\frac{1}{\pi} \operatorname{Im} \Pi^{R}\left(P, P ; k, k^{\prime} ; E\right),
$$

where $P$ is the external field operator. The relativistic RPA polarization operator is obtained by solving the Bethe-Salpeter equation[19],

$$
\begin{aligned}
& \Pi\left(Q, Q^{\prime} ; \mathbf{k}, \mathbf{k}^{\prime}, E\right)=\Pi_{0}\left(Q, Q^{\prime} ; \mathbf{k}, \mathbf{k}^{\prime}, E\right) \\
& -\sum_{i} g_{i}^{2} \int d^{3} k_{1} d^{3} k_{2} \Pi_{0}\left(Q, \Gamma^{i} ; \mathbf{k}, \mathbf{k}_{1}, E\right) \\
& D_{i}\left(\mathbf{k}_{1}, \mathbf{k}_{2}, E\right) \Pi\left(\Gamma_{i}, Q^{\prime} ; \mathbf{k}_{2}, \mathbf{k}^{\prime}, E\right),
\end{aligned}
$$

where the $\Gamma_{i}$ 's and $D_{i}$ 's are the vertex couplings and the corresponding meson propagators, respectively, $Q$ and $Q^{\prime}$ represent the external or vertex operators. Generally, the operators of spin-isospin excitations are

$$
P_{ \pm}=\frac{1}{\sqrt{2}} \sum_{i}^{A} r_{i}^{L} Y_{L \mu}\left(\hat{r}_{i}\right)\left(\boldsymbol{\sigma} \tau_{ \pm}\right)_{i},
$$

where $\tau_{ \pm}=\left(\tau_{x} \pm i \tau_{y}\right) / \sqrt{2}$ and $L=0$ for the GT operator. The operator is usually multiplied by $\gamma_{0}$ for vector density excitations.

The model-independent Ikeda sum rule[12] is expressed as

$$
\left\langle 0\left|P_{+} P_{-}\right| 0\right\rangle-\left\langle 0\left|P_{-} P_{+}\right| 0\right\rangle=3(N-Z),
$$

where $|0\rangle$ is either the nuclear correlated or uncorrelated ground state.

The vertex operators corresponding to $\pi$ - and $\rho$-coupling are

$$
\begin{aligned}
\Gamma_{\pi} \Gamma_{\pi} & =\gamma_{5} \gamma_{\mu} q^{\mu}(1) \gamma_{5} \gamma_{\mu} q^{\mu}(2) \boldsymbol{\tau}_{1} \boldsymbol{\tau}_{2}, \\
\Gamma_{\rho \mu} \Gamma_{\rho}^{\mu} & =\gamma_{\mu}(1) \gamma^{\mu} \boldsymbol{\tau}_{1} \boldsymbol{\tau}_{2} \\
& =\left[\gamma_{0}(1) \gamma_{0}(2)-\gamma_{0} \gamma_{5} \boldsymbol{\sigma}(1) \gamma_{0} \gamma_{5} \boldsymbol{\sigma}(2)\right] \boldsymbol{\tau}_{1} \boldsymbol{\tau}_{2},
\end{aligned}
$$


with the corresponding meson propagators

$$
\begin{aligned}
& D_{\pi}=\left(\frac{f_{\pi}}{m_{\pi}}\right)^{2} \frac{1}{(2 \pi)^{3}} \frac{1}{q^{2}-m_{\pi}^{2}+i \eta} \\
& D_{\rho}=-\frac{g_{\rho}^{2}}{(2 \pi)^{3}} \frac{1}{q^{2}-m_{\rho}^{2}+i \eta}
\end{aligned}
$$

The vertex operator of the Landau-Migdal force introduced in Eq.(2) is

$$
\Gamma_{\mu} \Gamma^{\mu}=\left[\gamma_{0} \gamma_{5}(1) \gamma_{0} \gamma_{5}(2)-\gamma_{0} \boldsymbol{\sigma}(1) \gamma_{0} \boldsymbol{\sigma}(2)\right] \boldsymbol{\tau}_{1} \boldsymbol{\tau}_{2}
$$

The time component and the current part have to be calculated separately. The propagator for the contact interaction term in the Lagrangian is

$$
D_{g^{\prime}}=\frac{g^{\prime}}{(2 \pi)^{3}}\left(\frac{f_{\pi}}{m_{\pi}}\right)^{2}
$$

The multipole expansion in momentum space is performed and the integrals of the angular parts can be carried out. The retarded unperturbed polarization operator with a fixed angular momentum $J$ in the momentum space is written as

$$
\begin{aligned}
& \Pi_{0}^{J}\left(Q, Q^{\prime} ; k, q ; E\right)=-\frac{(4 \pi)^{2}}{2 J+1}\left\{\sum_{p h}(-)^{j_{p}+j_{h}+1}\right. \\
& {\left[\frac{\left\langle\bar{\psi}_{h}\left\|\mathcal{Q}^{\dagger}\right\| \psi_{p}\right\rangle\left\langle\bar{\psi}_{p}\left\|\mathcal{Q}^{\prime}\right\| \psi_{h}\right\rangle}{\left(\varepsilon_{h}-\varepsilon_{p}\right)+E+i \eta}+\frac{\left\langle\bar{\psi}_{p}\left\|\mathcal{Q}^{\dagger}\right\| \psi_{h}\right\rangle\left\langle\bar{\psi}_{h}\left\|\mathcal{Q}^{\prime}\right\| \psi_{p}\right\rangle}{\left(\varepsilon_{h}-\varepsilon_{p}\right)-E-i \eta}\right]} \\
& -\sum_{\bar{\alpha} h}(-)^{j \bar{\alpha}^{+} j_{h}+1}\left[\frac{\left\langle\bar{\psi}_{\bar{\alpha}}\left\|\mathcal{Q}^{\dagger}\right\| \psi_{h}\right\rangle\left\langle\bar{\psi}_{h}\left\|\mathcal{Q}^{\prime}\right\| \psi_{\bar{\alpha}}\right\rangle}{\left(\varepsilon_{\bar{\alpha}}-\varepsilon_{h}\right)+E+i \eta}\right. \\
& \left.\left.+\frac{\left\langle\bar{\Psi}_{h}\left\|\mathcal{Q}^{\dagger}\right\| \psi_{\bar{\alpha}}\right\rangle\left\langle\bar{\psi}_{\bar{\alpha}}\left\|\mathcal{Q}^{\prime}\right\| \psi_{h}\right\rangle}{\left(\varepsilon_{\bar{\alpha}}-\varepsilon_{h}\right)-E-i \eta}\right]\right\}
\end{aligned}
$$

where $h, p$ and $\bar{\alpha}$ correspond to the occupied states in the Fermi sea, positive energy unoccupied states and negative energy states in the Dirac sea, respectively. The advantage of solving the Bethe-Salpeter equation in the momentum space is to allow the inclusion of as many configurations as required. Indeed, the numerical work amounts to invert matrices the size of which depends on the number of mesh points chosen in momentum space and the number or vertex operators. Therefore, increasing the number of configurations does not affect substantially the numerical effort in contrast to the matrix diagonalization method.

The external operator $\mathcal{P}$ after multipole expansion can be expressed as

$$
\mathcal{P}=\gamma_{0} r^{J-1}\left[Y_{J-1} \otimes \sigma\right]_{J}
$$


The vertex operator produced by the pion is

$$
\begin{aligned}
\mathcal{Q}^{\pi}= & -\gamma_{0} \gamma_{5} q_{0} Y_{J} j_{J}(k r)-\sum_{L=J \pm 1} i^{L+J} q \sqrt{2 L+1} \\
& \left(\begin{array}{ccc}
L & 1 & J \\
0 & 0 & 0
\end{array}\right) \gamma_{0}\left[Y_{L} \otimes \sigma\right]_{J} j_{L}(k r) .
\end{aligned}
$$

The time component of the operator produced by the vector part of the $\rho$ vanishes and only its space component contributes and leaves a minus sign on the propagator:

$$
\mathcal{Q}^{\rho}=\gamma_{0} \gamma_{5}\left[Y_{J} \otimes \sigma\right]_{J} j_{J}(k r)
$$

The time component of the vertex operator produced by the Landau-Migdal force is:

$$
\mathcal{Q}_{0}^{g^{\prime}}=\gamma_{0} \gamma_{5} Y_{J}(\hat{\mathbf{r}}) j_{J}(k r)
$$

Its space components are:

$$
\mathcal{Q}_{J \pm 1}^{g^{\prime}}=\gamma_{0}\left[Y_{J \pm 1} \otimes \sigma\right]_{J} j_{J \pm 1}(k r)
$$

which produces also a minus sign on the propagator. The isospin part of the operators is multiplied by $\tau_{+} / \sqrt{2}$. There are in total 5 operators for $\mathcal{Q}$, therefore the polarization $\Pi^{J}\left(Q, Q^{\prime}\right)$ is a $5 \times 5$ matrix.

The single-particle energies and wave functions are solutions of the self-consistent RMF equations obtained with the starting effective Lagrangian. A nucleon state can be specified by a set of quantum numbers $\alpha=\left(n_{a}, l_{a}, j_{a}, m_{a}\right) \equiv\left(a, m_{a}\right)$, where $q_{a}=-1$ and +1 for neutron and proton states, respectively. The nucleon wave function with energy $\varepsilon_{\alpha}$ is written as

$$
\psi_{\alpha}=\frac{1}{r}\left(\begin{array}{c}
i G_{a}(r) \\
F_{a}(r) \boldsymbol{\sigma} \cdot \hat{\mathbf{r}}
\end{array}\right) \mathcal{Y}_{\alpha}(\hat{\mathbf{r}}) \chi\left(q_{a}\right) .
$$

$\chi\left(q_{a}\right)$ and $\mathcal{Y}_{\alpha}$ being the isospinor and spin-spherical harmonic function, respectively.

The charge-exchange excitations flip the isospin and pick up only neutron-proton or protonneutron pairs. The reduced matrix element for the external spin-isospin excitation is

$$
\begin{aligned}
& \left\langle\bar{\psi}_{h}\|\mathcal{P}\| \psi_{p}\right\rangle=\int\left(G_{h} G_{p}\left\langle h\left\|T_{J-1} J\right\| p\right\rangle\right. \\
& \left.+F_{h} F_{p}\left\langle\bar{h}\left\|T_{J-1}\right\| \bar{p}\right\rangle\right) r^{J-1} d r
\end{aligned}
$$

where $\bar{h} \equiv n_{h} \bar{l}_{h} j_{h}$, and $\bar{l}$ is the orbital angular momentum of the lower component in the Dirac spinor. Here, we have introduced $\left\langle h\left\|T_{L J}\right\| p\right\rangle=\left\langle n_{h} l_{h} j_{h}\left\|\left[Y_{L} \otimes \sigma\right]_{J}\right\| n_{p} l_{p} j_{p}\right\rangle[20]$. The reduced matrix 
elements of the operators (14,17) are

$$
\begin{aligned}
& \left\langle\bar{\psi}_{h}\left\|\mathcal{Q}^{\pi}\right\| \psi_{p}\right\rangle=q_{0} \int\left(G_{h} F_{p}-F_{h} G_{p}\right) j_{J}(k r) d r \\
& \left\langle n_{h} l_{h} j_{h}\left\|Y_{J}\right\| n_{p} \bar{l}_{p} j_{p}\right\rangle+q \sum_{L=J \pm 1} \frac{f(L)}{\sqrt{2 L+1}} \\
& \times \int\left(G_{h} G_{p}\left\langle h\left\|T_{L J}\right\| p\right\rangle+F_{h} F_{p}\left\langle\bar{h}\left\|T_{L J}\right\| \bar{p}\right\rangle j_{L}(k r) d r\right.
\end{aligned}
$$

where $f(L)=J+1$ for $L=J+1$ and $J$ for $L=J-1$,

$$
\begin{gathered}
\left\langle\bar{\psi}_{h}\left\|\mathcal{Q}^{\rho}\right\| \psi_{p}\right\rangle=\int\left(G_{h} F_{p}\left\langle h\left\|T_{J J}\right\| \bar{p}\right\rangle\right. \\
\left.-F_{h} G_{p}\left\langle\bar{h}\left\|T_{J J}\right\| p\right\rangle\right) j_{J} d r \\
\left\langle\bar{\psi}_{h}\left\|\mathcal{Q}_{0}^{g^{\prime}}\right\| \psi_{p}\right\rangle=\int_{\left\langle n_{h} l_{h} j_{h}\left\|Y_{J}\right\| n_{p} \bar{l}_{p} j_{p}\right\rangle,}\left(G_{h} F_{p}-F_{h} G_{p}\right) j_{J}(k r) d r \\
\left\langle\bar{\psi}_{h}\left\|\mathcal{Q}_{J \pm 1}^{g^{\prime}}\right\| \psi_{p}\right\rangle=\int\left(G_{h} G_{p}\left\langle h\left\|T_{J \pm 1}\right\| p\right\rangle\right. \\
\left.+F_{h} F_{p}\left\langle\bar{h}\left\|T_{J \pm 1}\right\| \bar{p}\right\rangle\right) j_{J \pm 1}(k r) d r .
\end{gathered}
$$

We calculate the $L=0 \mathrm{GT}$ excitations for the double closed shell nuclei ${ }^{48} \mathrm{Ca},{ }^{90} \mathrm{Zr}$ and ${ }^{208} \mathrm{~Pb}$, which have been extensively investigated experimentally. The ground state wave functions of those nuclei are calculated in the RMF with the parameter set NL3 7]. The continuous singleparticle spectrum is discretized in a harmonic oscillator basis. An averaging parameter $\Delta=2$ $\mathrm{MeV}$ is used to smooth out the response functions, which is performed by replacing the excitation energy $E+i \eta$ in Eq.(8) by $E+i \Delta / 2$. The residual p-h interaction is produced through the isovector mesons and the Landau-Migdal force in the Lagrangian Eqs.(10.2), where $g^{\prime}=0.6$ and the standard values $f_{\pi}^{2} / 4 \pi=0.08, m_{\pi}=138 \mathrm{MeV}$ are adopted. All other coupling constants are those of NL3.

The calculated Hartree and correlated GT strengths for ${ }^{48} \mathrm{Ca},{ }^{90} \mathrm{Zr}$ and ${ }^{208} \mathrm{~Pb}$ are shown in Fig.1. The dashed and solid curves correspond to the Hartree and RPA results, respectively. The main contributions to the GT excitation are from the neutrons at the Fermi surface excited to the unoccupied proton states with the same orbital angular momentum. For instance, the main p-h configurations are: $\left(\pi 1 g_{9 / 2}\left(\nu 1 g_{9 / 2}\right)^{-1}\right)$ with $\varepsilon_{p h}=6.3 \mathrm{MeV}$ and $\left(\pi 1 g_{7 / 2}\left(\nu 1 g_{9 / 2}\right)^{-1}\right)$ with $\varepsilon_{p h}=13.9 \mathrm{MeV}$ in ${ }^{90} \mathrm{Zr} ;\left(\pi 1 f_{7 / 2}\left(\nu 1 f_{7 / 2}\right)^{-1}\right), \varepsilon_{p h}=0.3 \mathrm{MeV}$ and $\left(\pi 1 f_{5 / 2}\left(\nu 1 f_{7 / 2}\right)^{-1}\right), \varepsilon_{p h}=$ $8.4 \mathrm{MeV}$ in ${ }^{48} \mathrm{Ca}$, which exhibit peaks in the Hartree strengths. There are more contributing configurations in ${ }^{208} \mathrm{~Pb}$. The collectivity effect is mainly due to the Landau-Migdal term of the 


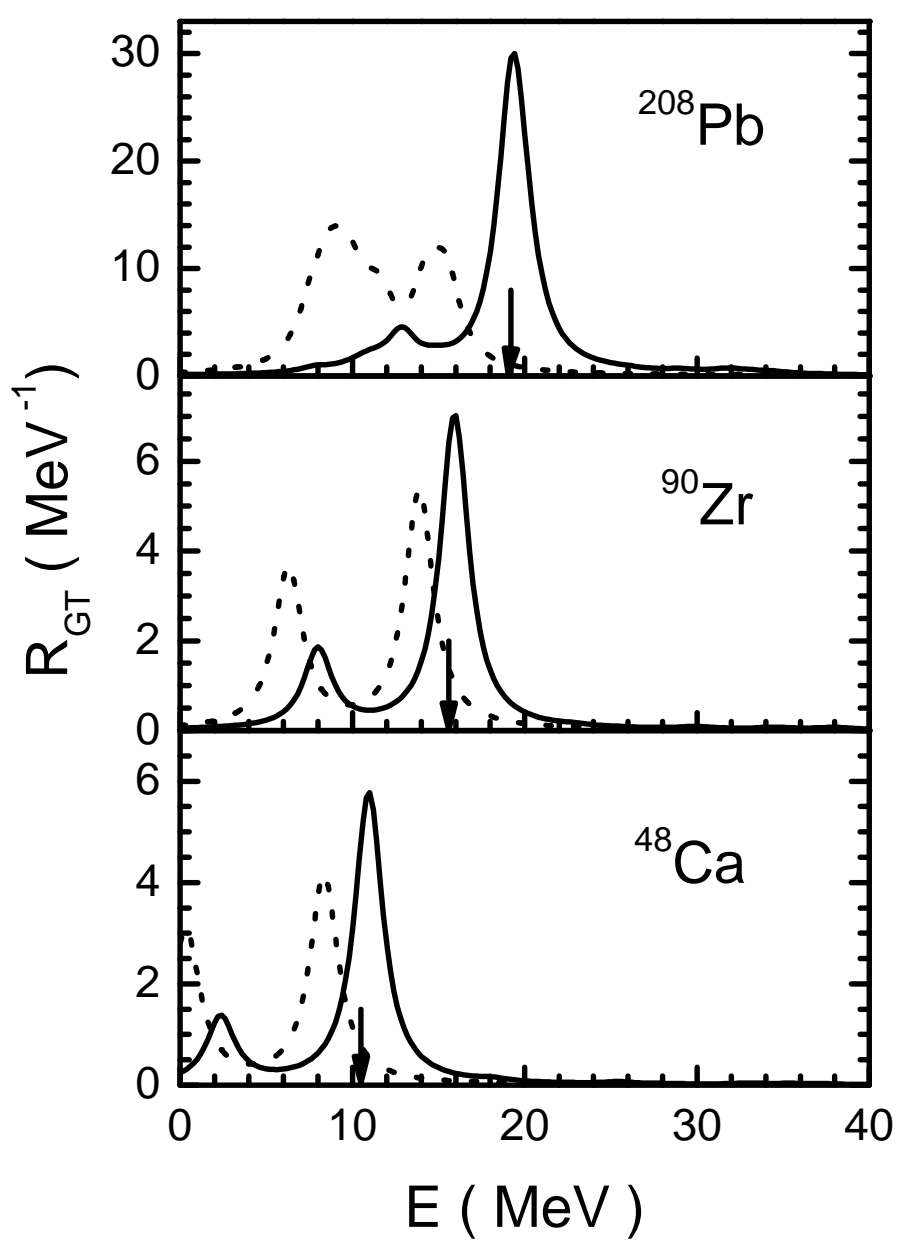

FIG. 1: GT response functions for ${ }^{48} \mathrm{Ca},{ }^{90} \mathrm{Zr}$ and ${ }^{208} \mathrm{~Pb}$. The GT strengths are calculated in the relativistic RPA with the parameter set NL3. The solid and dashed curves correspond to the RPA and Hartree strengths, respectively. Experimental energies are shown by the arrows.

residual interaction. It results in an upward shift of the strength. The centroid energies of the main peaks in Hartree and in RPA are listed in Table 1. A good agreement with the experimental data is observed when $g^{\prime}=0.6$ is chosen. The total strengths up to $60 \mathrm{MeV}$ exhaust the Ikeda sum rule by about 92-94 \%. From Table 1 it can also be seen that the total GT strength below $60 \mathrm{MeV}$ is similar in Hartree and in RPA. In ref.[17] it was also found that the GT quenching is about $12 \%$ in infinite matter and $6 \%$ in nuclei calculated in Hartree approximation. The rest of the strength is connected with the effects of the Dirac sea states. NN pairs involving states in the Fermi sea and negative energy states can also carry GT strength, if one considers backward-going graphs and the no-sea approximation.

In summary, we have investigated in finite nuclei the nuclear spin-isospin excitations, espe- 
TABLE I: GT energies of ${ }^{48} \mathrm{Ca},{ }^{90} \mathrm{Zr}$ and ${ }^{208} \mathrm{~Pb} . \bar{E}$ corresponds to the centroid energies of theoretical results with $g^{\prime}=0.6$. The percentages of Ikeda sum rule are calculated up to $E \leq 60 \mathrm{MeV}$. The experimental data are taken from Refs. 10, 21, 22]

\begin{tabular}{cccccc}
\hline \hline & \multicolumn{3}{c}{ Theoretical Results } & \multicolumn{2}{c}{ Experiment } \\
\cline { 2 - 6 } Nuclei & $\bar{E}(\mathrm{MeV})$ & Strength \% of sum rule & Energy & Strength \\
& $\mathrm{RRPA}$ & Hartree & RRPA & $(\mathrm{MeV})$ & $\%$ of sum rule \\
\hline${ }^{48} \mathrm{Ca}$ & 10.1 & 93.0 & 93.8 & 10.5 & 35 \\
${ }^{90} \mathrm{Zr}$ & 15.4 & 92.3 & 93.2 & $15.6 \pm 0.3$ & $90 \pm 5$ \\
${ }^{208} \mathrm{~Pb}$ & 18.9 & 91.6 & 92.6 & $19.2 \pm 0.2$ & $60 \sim 70$ \\
\hline \hline
\end{tabular}

cially the GT resonance in a fully relativistic RPA. A relativistic form of the Landau-Migdal contact interaction in the spin-isospin channel is adopted, which reproduces in the non-relativistic limit the excitation energy of the giant GT resonance in infinite matter 17]. The GT resonances in ${ }^{48} \mathrm{Ca},{ }^{90} \mathrm{Zr}$ and ${ }^{208} \mathrm{~Pb}$ are investigated. It is found that the RPA strengths up to $60 \mathrm{MeV}$ exhaust the Ikeda sum rule by only about 93\%. The missing fraction is taken by the pairs formed between states in the Fermi sea and Dirac sea. This quenching mechanism is specific of the relativistic description of the GT mode.

\section{Acknowledgments}

This work is partly supported by the National Natural Science Foundation of China under Grant Nos 10275094, 10075080 and 10235020, and the Major State Basic Research Development Programme of China under Contract No G2000077400. One of the authors(T.S.) would like to thank Professor H. Kurasawa for useful discussions.

[1] P. Ring, Prog. Part. Nucl. Phys. 37 (1996) 197.

[2] J.F. Dawson and R.J. Furnstahl, Phys. Rev. C 42 (1990) 2009.

[3] C.J. Horowitz and J. Piekarewicz, Nucl. Phys. A 511 (1990) 461.

[4] Z. Y. Ma Commn. Theor. Phys. 32 (1999) 493.

[5] Z. Y. Ma, N. Van Giai, A. Wandelt, D. Vretenar and P. Ring, Nucl. Phys. A 686 (2001) 173.

[6] D. Vretenar, Nucl. Phys. A 649 (1999) 29c. 
[7] G. A. Lalazissis et al., Phys. Rev. C 55 (1997) 540.

[8] P. Ring, Z. Y. Ma, N. Van Giai, D. Vretenar, and A. Wandelt, L. G. Cao, Nucl. Phys. A 694 (2001) 249.

[9] Z. Y. Ma, A. Wandelt, N. Van Giai, D. Vretenar, P. Ring, and L. G. Cao Nucl. phys. A 703 (2002) 222 .

[10] T. Wakasa et al., Phys. Rev. C 55 (1997) 2909.

[11] T. Wakasa, H. Sakai, S. Fujita, T. Noanaka et al., Nucl. Phys. A 687 (2001) 26c; and to be published in Nucl. Phys. A.

[12] K. Ikeda, S. Fujii and J. I. Fujita, Phys. Lett. 3 (1963) 271.

[13] G.F. Bertsch and I. Hamamoto, Phys. Rev. C 26 (1982) 1323.

[14] E. Oset and M Rho, Phys. Rev. Lett. 42 (1979) 47.

[15] C. De Conti, A. P. Galeão, E. Krmpotié, Phys. lett. B 444 (1998) 14.

[16] C. De Conti, A. P. Galeão, E. Krmpotié, Phys. lett. B 494 (2000) 46.

[17] H. Kurasawa, T. Suzuki and N. Van. Giai, nucl-th/0301074, Phys. Rev. Lett. (2003, in press); nucl-th/0306080

[18] C. J. Horowitz and J. Piekarewicz, Phys. Rev. C 50 (1994) 2540.

[19] Z. Y. Ma, N. Van Giai and H. Toki and M. L'Huillier, Phys. Rev. C 55 (1997) 2385.

[20] D. M. Brink and G. R. Satchler, Angular Momentum (London, Oxford University Press, 1962).

[21] B. D. Anderson et al., Phys. Rev. C 31 (1985)1161.

[22] H. Akimune et al., Phys. Rev. C 52 (1995) 604. 